PROCEEDINGS OF THE

AMERICAN MATHEMATICAL SOCIETY

Volume 125, Number 8, August 1997, Pages 2395-2399

S 0002-9939(97)03991-9

\title{
WAVELET DECOMPOSITIONS OF FOURIER MULTIPLIERS
}

\author{
EARL BERKSON, MACIEJ PALUSZYŃSKI, AND GUIDO WEISS
}

(Communicated by J. Marshall Ash)

\begin{abstract}
We show that in terms of its weak* topology, the space of Fourier multipliers for $L^{p}(\mathbb{R}), 1<p<\infty$, can be decomposed by band-limited wavelets belonging to the Schwartz class.
\end{abstract}

\section{INTRODUCTION AND NOTATION}

A function $w \in L^{2}(\mathbb{R})$ is called a wavelet provided that the sequence $\left\{w_{j, k}\right\}_{j \in \mathbb{Z}, k \in \mathbb{Z}}$ defined by $w_{j, k} \equiv 2^{j / 2} w\left(2^{j} x-k\right)$ is an orthonormal basis for $L^{2}(\mathbb{R})$. It is wellknown that under these circumstances $\left\{w_{j, k}\right\}_{j \in \mathbb{Z}, k \in \mathbb{Z}}$ often serves as a basis for numerous spaces of interest in analysis. In the spirit of this theme, the present note applies the theory of wavelets to Fourier analysis per se by showing that, in an appropriate sense, certain wavelets belonging to the Schwartz class $\mathcal{S}$ on $\mathbb{R}$ decompose the Fourier multipliers for $L^{p}(\mathbb{R}), 1<p<\infty$. In order to state and establish our results (see $\S 2$ ), we begin by recalling some essential background items and fixing notation. Suppose that $1 \leq p<\infty, q$ is the index conjugate to $p$, and $\Gamma$ is the dual group of a locally compact abelian group $G$. The space $M_{p}(\Gamma)$ of Fourier multipliers for $L^{p}(G)$ consists of the functions $\psi \in L^{\infty}(\Gamma)$ such that $T_{\psi}$ is a continuous linear mapping of $L^{p}(G)$ into $L^{p}(G)$, where $T_{\psi}$ is given by the formula $T_{\psi} f=(\psi \hat{f})^{\vee}$, for all $f \in L^{2}(\mathbb{R}) \cap L^{p}(\mathbb{R}) . M_{p}(\Gamma)$ is a Banach algebra under pointwise operations and the norm $\|\psi\|_{M_{p}(\Gamma)} \equiv\left\|T_{\psi}\right\|_{p}$. As shown in [5, Theorem 1], when $1<p<\infty, M_{p}(\Gamma)$ can be identified (via a surjective linear isometry) with the dual space of $A_{p}(G)$. The Banach space $A_{p}(G)$ is composed of the functions $f$ which can be written in the form

$$
f=\sum_{n=1}^{\infty} f_{n} * g_{n}
$$

where $\left\{f_{n}\right\}_{n=1}^{\infty} \subseteq L^{p}(G),\left\{g_{n}\right\}_{n=1}^{\infty} \subseteq L^{q}(G)$, and $\sum_{n=1}^{\infty}\left\|f_{n}\right\|_{p}\left\|g_{n}\right\|_{q}<\infty$. The norm $\|f\|_{A_{p}(G)}$ is the infimum of the sums $\sum_{n=1}^{\infty}\left\|f_{n}\right\|_{p}\left\|g_{n}\right\|_{q}$ corresponding to the representations for $f$ in (1). By starting with an arbitrary representation (1) for $f \in A_{p}(G)$, we can specify the duality relationship between $M_{p}(\Gamma)$ and $A_{p}(G)$ by writing $\psi[f]=\sum_{n=1}^{\infty}\left(\left(T_{\psi} f_{n}\right) * g_{n}\right)(0)$. Furthermore, if $F \in L^{1}(\Gamma)$, and $g(x) \equiv \int_{\Gamma} F(\gamma) \gamma(x) d \gamma$, then $g \in A_{p}(G)$, and $\psi[g]=\int_{\Gamma} \psi(\gamma) F(\gamma) d \gamma$. (It follows

Received by the editors March 4, 1996.

1991 Mathematics Subject Classification. Primary 42A45, 42C15.

The work of the first and third authors was supported by separate grants from the National Science Foundation (U.S.A.).

The second author wishes to thank DARPA for its support. 
from this, by a standard Hahn-Banach argument, that $L^{1}(\Gamma)^{\wedge}$ is norm dense in $A_{p}(G)$-a fact also shown in the course of [5, proof of Theorem 1].) The wavelet decompositions in $M_{p}(\mathbb{R})$ described below will be formulated in terms of the weak* topology $\sigma\left(M_{p}(\mathbb{R}), A_{p}(\mathbb{R})\right)$.

Let $\Omega$ be the class of wavelets $w \in \mathcal{S}$ such that: (i) the support of $\widehat{w}(t) \equiv$ $\int_{\mathbb{R}} w(x) e^{-i x t} d x$ has the form $\{t \in \mathbb{R}: \pi-\varepsilon \leq|t| \leq 2(\pi+\varepsilon)\}$, where $\varepsilon$ belongs to $(0, \pi / 3]$; and (ii) $|\widehat{w}|$ is an even function. The most general wavelet in $L^{2}(\mathbb{R})$ satisfying (i) and (ii) has been characterized in [4, Theorem (3.1)]. The study of the class $\Omega$ has its origins in [6], where the constructions furnished an element of $\Omega$ such that $\varepsilon$ in (i) has the value $\frac{\pi}{3}$. Subsequently it was shown in [3, $\left.\S \S 1-3\right]$ that for each $\varepsilon$ belonging to $(0, \pi / 3]$ there is a corresponding wavelet $w \in \Omega$ such that (i) holds. Now suppose that $\Lambda \in \Omega$. By virtue of $\left[2\right.$, Theorem 1.3] the wavelet basis $\left\{\Lambda_{j, k}\right\}_{j \in \mathbb{Z}, k \in \mathbb{Z}}$ for $L^{2}(\mathbb{R})$ arises from a multiresolution analysis which has an associated scaling function $\Delta \in \mathcal{S}$ such that $\widehat{\Delta}$ is compactly supported. As usual, we choose $\Delta$ so that $\int_{\mathbb{R}} \Delta(x) d x=1$. (This notation for $\Lambda \in \Omega$ and $\Delta \in \mathcal{S}$ will remain in effect henceforth.) The self-adjoint projection operators $\left\{E_{j}\right\}_{j=-\infty}^{\infty}$ corresponding to the multiresolution analysis have the following familiar description:

$$
\left(E_{j} f\right)(x)=2^{j} \int_{\mathbb{R}} K\left(2^{j} x, 2^{j} y\right) f(y) d y=\sum_{k=-\infty}^{\infty}\left\langle f, \Delta_{j, k}\right\rangle \Delta_{j, k}(x), \quad \text { for all } x \in \mathbb{R},
$$

where $\Delta_{j, k}(x) \equiv 2^{j / 2} \Delta\left(2^{j} x-k\right), K(x, y) \equiv \sum_{k=-\infty}^{\infty} \Delta_{0, k}(x) \overline{\Delta_{0, k}}(y)$, and $\left\langle f, \Delta_{j, k}\right\rangle$ $=\int_{\mathbb{R}} f(y) \overline{\Delta_{j, k}}(y) d y$. Since

$$
\sum_{k=-\infty}^{\infty}\left|\Delta_{0, k}(x) \Delta_{0, k}(y)\right| \leq C_{m}(1+|x-y|)^{-m}, \quad \text { for all } x \in \mathbb{R},, y \in \mathbb{R}, \text { and } m \in \mathbb{N} \text {, }
$$

the scope of (2) is not limited to the functions $f$ belonging to the initial space $L^{2}(\mathbb{R})$ : for $1 \leq p \leq \infty,(2)$ also defines $E_{j}$ as a continuous linear mapping of $L^{p}(\mathbb{R})$ into $L^{p}(\mathbb{R})$ such that $E_{j}^{2}=E_{j}$. Analogous assertions are valid for the idempotent operators $\left\{D_{j}\right\}_{j=-\infty}^{\infty}$ defined on $L^{p}(\mathbb{R}), 1 \leq p \leq \infty$, by $D_{j}=E_{j+1}-E_{j}$. Specifically, we have

$$
\left(D_{j} f\right)(x)=2^{j} \int_{\mathbb{R}} \widetilde{K}\left(2^{j} x, 2^{j} y\right) f(y) d y=\sum_{k=-\infty}^{\infty}\left\langle f, \Lambda_{j, k}\right\rangle \Lambda_{j, k}(x), \quad \text { for all } x \in \mathbb{R},
$$

where $\widetilde{K}(x, y) \equiv \sum_{k=-\infty}^{\infty} \Lambda_{0, k}(x) \overline{\Lambda_{0, k}}(y)$ and

$$
\sum_{k=-\infty}^{\infty}\left|\Lambda_{0, k}(x) \Lambda_{0, k}(y)\right| \leq C_{m}(1+|x-y|)^{-m} \quad \text { for all } x \in \mathbb{R}, y \in \mathbb{R} \text {, and } m \in \mathbb{N} \text {. }
$$

We shall also benefit from the relations of scale: $E_{j}=\delta_{2^{j}} E_{0} \delta_{2^{-j}}$ and $D_{j}=$ $\delta_{2^{j}} D_{0} \delta_{2^{-j}}$, where $\left(\delta_{\alpha} f\right)(x) \equiv f(\alpha x)$. Notice that $D_{m} D_{n}=0$ for $m \neq n$, since $j \leq k$ implies $E_{j} E_{k}=E_{k} E_{j}=E_{j}$ on $L^{p}(\mathbb{R}), 1 \leq p \leq \infty$.

The following theorem furnishes the starting point for the links between wavelets and multipliers developed in $\S 2$. 
Theorem 1 ([1, Theorem (5.6)]). Suppose that $h \in L^{1}(\mathbb{R})$ is continuous on $\mathbb{R}$, $B \in(0, \infty)$, the support of $\widehat{h}$ is a subset of $[-B, B], \widehat{h}$ is absolutely continuous on $\mathbb{R}$, and the derivative of $\widehat{h}$ belongs to $L^{2}(\mathbb{R})$. If $1 \leq p<\infty$ and $\phi \in M_{p}(\mathbb{Z})$, then $\mathcal{W}_{\phi, h} \in M_{p}(\mathbb{R})$, where $\mathcal{W}_{\phi, h}(x)=\sum_{k=-\infty}^{\infty} \phi(k) h(x-k)$ for all $x \in \mathbb{R}$. Moreover

$$
\left\|\mathcal{W}_{\phi, h}\right\|_{M_{p}(\mathbb{R})} \leq 2^{N+(\max \{p, q\})^{-1}} \delta(h)\|\phi\|_{M_{p}(\mathbb{Z})},
$$

where $N$ is the least non-negative integer such that $B 2^{-N} \leq \pi / 2$, and

$$
\delta(h)=\sup _{x \in \mathbb{R}} \sum_{k=-\infty}^{\infty}|h(x-k)|<\infty .
$$

$\mathcal{W}_{\phi, h}$ can be extended to $\mathbb{C}$ as an entire function of exponential type.

\section{WAVELET DECOMPOSITIONS IN $M_{p}(\mathbb{R})$}

Our main results are stated in the following two theorems. Observe beforehand that since $M_{p}(\mathbb{R}) \subseteq L^{\infty}(\mathbb{R})$, the operators $D_{j}$ and $E_{j}, j \in \mathbb{Z}$, map $M_{p}(\mathbb{R})$ into $L^{\infty}(\mathbb{R})$.

Theorem 2. Suppose that $1<p<\infty$ and $\Lambda \in \Omega$. Then for each $j \in \mathbb{Z}, D_{j}$ and $E_{j}$ are continuous idempotent linear transformations of $M p(\mathbb{R})$ into $M_{p}(\mathbb{R})$. The image $D_{j}\left(M_{p}(\mathbb{R})\right)$ is the weak ${ }^{*}$-closed subspace of $M_{p}(\mathbb{R})$ generated by $\left\{\Lambda_{j, k}\right\}_{k=-\infty}^{\infty}$. If $\psi \in$ $M_{p}(\mathbb{R})$, then $\sum_{k=-N}^{N}\left\langle\psi, \Lambda_{j, k}\right\rangle \Lambda_{j, k}$ converges in the weak $k^{*}$ topology $\sigma\left(M_{p}(\mathbb{R}), A_{p}(\mathbb{R})\right)$ to $D_{j} \psi$, as $N \rightarrow \infty$. If, in addition, $\psi(t) \rightarrow 0$ as $|t| \rightarrow \infty$, then $\sum_{j=-N}^{N} D_{j} \psi$

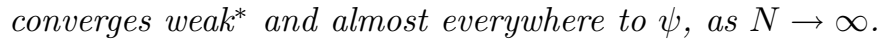

Theorem 3. Suppose that $1<p<\infty$ and $\Lambda \in \Omega$. Then the weak* ${ }^{*}$-closed subspace of $M_{p}(\mathbb{R})$ generated by $\left\{\Lambda_{j, k}\right\}_{j \in \mathbb{Z}, k \in \mathbb{Z}}$ is $M_{p}(\mathbb{R})$.

Proof of Theorem 2. Let $\psi \in M_{p}(\mathbb{R})$ and put $\Lambda^{\star}(t) \equiv \overline{\Lambda(-t)}$. Then, with the aid of De Leeuw's Restriction Theorem for Multipliers, we find that

$$
\left\|\left\{\left\langle\psi, \Lambda_{0, k}\right\rangle\right\}_{k=-\infty}^{\infty}\right\|_{M_{p}(\mathbb{Z})}=\left\|\left(\Lambda^{\star} * \psi\right) \mid \mathbb{Z}\right\|_{M_{p}(\mathbb{Z})} \leq\|\Lambda\|_{1}\|\psi\|_{M_{p}(\mathbb{R})} .
$$

In view of Theorem 1 this implies that $D_{0}$ is a continuous linear mapping of $M_{p}(\mathbb{R})$ into $M_{p}(\mathbb{R})$ (and similarly so is $E_{0}$ ). If $F \in L^{1}(\mathbb{R})^{\wedge}$, it follows by dominated convergence that $\left(\sum_{k=-N}^{N}\left\langle\psi, \Lambda_{0, k}\right\rangle \Lambda_{0, k}\right)[F] \rightarrow\left(D_{0} \psi\right)[F]$, as $N \rightarrow \infty$. For $N \in \mathbb{N}$, let $\chi_{N}: \mathbb{Z} \rightarrow \mathbb{C}$ be the characteristic function of $[-N, N] \cap \mathbb{Z}$. The M. Riesz Theorem, together with an application of Theorem 1 to $\left\{\left\langle\psi, \Lambda_{0, k}\right\rangle \chi_{N}(k)\right\}_{k=-\infty}^{\infty} \in$ $M_{p}(\mathbb{Z})$, shows that

$$
\sup _{N \in \mathbb{N}}\left\|\sum_{k=-N}^{N}\left\langle\psi, \Lambda_{0, k}\right\rangle \Lambda_{0, k}\right\|_{M_{p}(\mathbb{R})}<\infty .
$$

Since $L^{1}(\mathbb{R})^{\wedge}$ is dense in $A_{p}(\mathbb{R})$, it is clear from the foregoing observations that, as $N \rightarrow \infty$,

$$
\sum_{k=-N}^{N}\left\langle\psi, \Lambda_{0, k}\right\rangle \Lambda_{0, k} \rightarrow D_{0} \psi, \quad \text { in the weak }{ }^{*} \text { topology of } M_{p}(\mathbb{R}) .
$$


Conversely, let $\phi$ belong to the weak*-closed subspace $Q$ of $M_{p}(\mathbb{R})$ generated by $\left\{\Lambda_{0, k}\right\}_{k=-\infty}^{\infty}$, and put $\Phi=\phi-D_{0} \phi \in Q$. Since

$$
\Psi[\overline{\hat{f}}]=\langle\Psi, f\rangle, \quad \text { for } \Psi \in M_{p}(\mathbb{R}), f \in L^{1}(\mathbb{R}),
$$

it is clear from the orthonormality of $\left\{\Lambda_{j, k}\right\}_{j \in \mathbb{Z}, k \in \mathbb{Z}}$ that $\left\langle\phi, \Lambda_{j, k}\right\rangle=0$, for $j \in$ $\mathbb{Z} \backslash\{0\}, k \in \mathbb{Z}$. Applying this result to $\Phi \in Q$ in place of $\phi$, we readily infer that $\left\langle\Phi, \Lambda_{j, k}\right\rangle=0$ for all $j \in \mathbb{Z}$ and all $k \in \mathbb{Z}$. In view of the well-known fact that $\left\{\Lambda_{j, k}\right\}_{j \in \mathbb{Z}, k \in \mathbb{Z}}$ generates $H^{1}(\mathbb{R})$, this shows that $\Phi$ is a constant. Because $\Phi \in Q$, another application of (4) gives $\langle\Phi, \Delta\rangle=0$. Hence the constant value of $\Phi$ is 0 , and so $\phi=D_{0} \phi$. After a change of scale from the level $j=0$ to the general level $j \in \mathbb{Z}$, it remains only to establish the last assertion of the theorem. For this purpose suppose first that $\psi$ is an arbitrary element of $M_{p}(\mathbb{R})$. The reasoning in [7, p. 33], with obvious modifications, shows that $\left(E_{j} \psi\right)(x) \rightarrow \psi(x)$ as $j \rightarrow \infty$, for every $x$ in the Lebesgue set of $\psi$. If we further assume that $\lim _{|t| \rightarrow \infty} \psi(t)=0$, then another elementary argument using the kernel $K$ in (2) shows that $\left\|E_{j} \psi\right\|_{\infty} \rightarrow 0$, as $j \rightarrow-\infty$. Since $\sum_{j=-N}^{N} D_{j}=E_{N+1}-E_{-N}$, the required a.e. convergence is evident, and the required weak* convergence follows with the aid of a dominated convergence argument.

Proof of Theorem 3. Suppose, to the contrary, that there is $f=\sum_{n=1}^{\infty} f_{n} * g_{n} \in$ $A_{p}(\mathbb{R})$ such that $\|f\|_{A_{p}(\mathbb{R})}=1$ and for all $j \in \mathbb{Z}, k \in \mathbb{Z}$

$$
0=\Lambda_{j, k}[f]=\sum_{n=1}^{\infty}\left(\left(\Lambda_{j, k}\right)^{\vee} *\left(f_{n} * g_{n}\right)\right)(0)=(2 \pi)^{-1} \int_{\mathbb{R}} f(t) \widehat{\Lambda}_{j, k}(t) d t .
$$

This implies that $f \equiv 0$ by virtue of the reasoning used for the final stage in the proof of [4, Theorem (3.1)], and consequently we have arrived at a contradiction.

(5) Remark. With obvious modifications, the reasoning used to establish Theorem 2 also shows that if $1<p<\infty, j \in \mathbb{Z}$, and $\psi \in M_{p}(\mathbb{R})$, then $\sum_{k=-N}^{N}\left\langle\psi, \Delta_{j, k}\right\rangle \Delta_{j, k}$ converges weak* to $E_{j} \psi$, as $N \rightarrow \infty$.

The next result shows that the operators $E_{j}$ and $D_{j}$ on $M_{p}(\mathbb{R})$ preserve weak* convergence.

Theorem 4. Suppose that $1<p<\infty$ and $\Lambda \in \Omega$. Then for $j \in \mathbb{Z}$ each of the operators $E_{j}$ and $D_{j}$ on $M_{p}(\mathbb{R})$ is the adjoint of a corresponding continuous linear operator mapping $A_{p}(\mathbb{R})$ into $A_{p}(\mathbb{R})$.

Proof. Denote by $C_{0}^{\infty}(\mathbb{R})$ the dense linear subspace of $A_{p}(\mathbb{R})$ consisting of all $f \in$ $C^{\infty}(\mathbb{R})$ such that $f$ has compact support. For $f \in C_{0}^{\infty}(\mathbb{R})$ and $k \in \mathbb{Z}$, we have

$$
\Delta_{0, k}[f]=\frac{1}{2 \pi} \int_{\mathbb{R}} \widehat{f}(t) \Delta(t-k) d t .
$$

Since $\widehat{f} \in \mathcal{S}$ and $\Delta \in \mathcal{S}$, standard estimates on the right-hand side of this equation prove that the sequence $\left\{\Delta_{0, k}[f]\right\}_{k=-\infty}^{\infty}$ is rapidly decreasing-that is, for each $m \in$ $\mathbb{N}$, there is a constant $C_{m}$ such that

$$
\left|\Delta_{0, k}[f]\right| \leq \frac{C_{m}}{|k|^{m}}, \quad \text { for all } k \in \mathbb{Z} \backslash\{0\} .
$$

Next suppose that $F \in L^{1}(\mathbb{R})$ and $t \in \mathbb{R}$. Let $F_{t}$ denote the translate of $F$ by $t$. By performing simple calculations which use the duality relationship between $A_{p}(\mathbb{R})$ 
and $M_{p}(\mathbb{R})$, we see that

$$
\left\|\left(F_{t}\right) \widehat{ }\right\|_{A_{p}(\mathbb{R})}=\left\|F^{\widehat{\imath}}\right\|_{A_{p}(\mathbb{R})} .
$$

It follows immediately from the foregoing discussion that if $f \in C_{0}^{\infty}(\mathbb{R})$, then the series

$$
\sum_{k=-\infty}^{\infty}\left(\Delta_{0, k}[f]\right) \overline{\widehat{\Delta}}_{0, k}
$$

converges absolutely in the space $A_{p}(\mathbb{R})$ to some vector $g$. Hence for each $\psi \in$ $M_{p}(\mathbb{R})$, we infer with the aid of $(4)$ that

$$
\psi[g]=\sum_{k=-\infty}^{\infty}\left(\Delta_{0, k}[f]\right)\left\langle\psi, \Delta_{0, k}\right\rangle .
$$

Applying Remark (5) (in the case $j=0$ ) to this, we get $\psi[g]=\left(E_{0} \psi\right)[f]$, and consequently $\|g\|_{A_{p}(\mathbb{R})} \leq K\|f\|_{A_{p}(\mathbb{R})}$, where $K$ denotes the norm of $E_{0}$ as a continuous linear map of $M_{p}(\mathbb{R})$ into $M_{p}(\mathbb{R})$. Invoking the density of $C_{0}^{\infty}(\mathbb{R})$ in $A_{p}(\mathbb{R})$, we can now readily deduce that the operator $E_{0}$ on $M_{p}(\mathbb{R})$ is the adjoint of a bounded linear transformation mapping $A_{p}(\mathbb{R})$ into $A_{p}(\mathbb{R})$. The assertion of the theorem regarding $E_{j}, j \in \mathbb{Z}$, follows at once by a change of scale, and then furnishes the desired conclusion for $D_{j}=E_{j+1}-E_{j}$.

In closing we observe that methods based on Theorem 1 similar to those used in the demonstration of Theorem 2 can be combined with the Closed Graph Theorem to give the following result.

Proposition. If $\Lambda \in \Omega$ and $1 \leq p<\infty$, then $D_{0}$ is an idempotent bounded linear transformation of $M_{p}(\mathbb{R})$ into $M_{p}(\mathbb{R})$. The mapping $\phi \in M_{p}(\mathbb{Z}) \mapsto \mathcal{W}_{\phi, \Lambda}$ is an injective bicontinuous linear transformation of $M_{p}(\mathbb{Z})$ onto $D_{0}\left(M_{p}(\mathbb{R})\right)$.

\section{REFERENCES}

1. N. Asmar, E. Berkson, and T.A. Gillespie, On Jodeit's multiplier extension theorems, Journal d'Analyse Math. 64 (1994), 337-345. MR 96j:42001

2. P. Auscher, Solution of two problems on wavelets,, J. Geometric Analysis 5 (1995), 181-236. MR 96g: 42016

3. P. Auscher, G. Weiss, and M.V. Wickerhauser, Local sine and cosine bases of Coifman and Meyer and the construction of smooth wavelets, "Wavelets: A Tutorial in Theory and Applications", Academic Press, New York, 1992, pp. 237-256. MR 93e:42042

4. A. Bonami, F. Soria, and G. Weiss, Band limited wavelets, J. Geometric Analysis 3 (1993), 543-578. MR 94k:42046

5. A. Figà-Talamanca, Translation invariant operators in $L^{p}$, Duke Math. J. 32 (1965), 495-501. MR 31:6095

6. P.G. Lemarié and Y. Meyer, Ondelettes et bases hilbertiennes, Rev. Mat. Iberoamericana 2 (1986), 1-18. CMP 19:04

7. Y. Meyer, Ondelettes et algorithmes concurrents, Actualités Scientifiques et Industrielles 1435, Hermann, Paris, 1992. MR 94g:42059

Department of Mathematics, University of Illinois, 1409 West Green St., Urbana, ILLINOIS 61801

Institute of Mathematics, University of Wroclaw, Wroclaw, Poland

Department of Mathematics, Washington University, St. Louis, Missouri 63130 